\title{
Study on Organic Light-Emitting Device with More Balanced Charge Transport
}

\author{
Jiun-Haw Lee ${ }^{\mathrm{a}}$, Tien-Chun Lin ${ }^{* a}$, Chi-Chih Liao ${ }^{\mathrm{b}}$, and F. H. Yang ${ }^{\mathrm{c}}$ \\ ${ }^{a}$ Graduate Institute of Electro-Optical Engineering and Department of Electrical Engineering, \\ National Taiwan University, No. 1, Sec. 4, Roosevelt Road, Taipei, Taiwan, R.O.C. \\ ${ }^{\mathrm{b}}$ RiTdisplay Corporation, Hsin-Chu Industrial Park, Hsin-Chu, Taiwan, R.O.C. \\ ${ }^{c}$ Aixtron Taiwan Corporation, Science-based Industrial Park, Hsin-Chu, Taiwan, R.O.C. \\ (E-mail) oeoled@yahoo.com.tw
}

\begin{abstract}
In this paper, we present the device performance of $\mathrm{N}^{4}, \mathrm{~N}^{4}$-Di-naphthalen-2-yl- $\mathrm{N}^{4}, \mathrm{~N}^{4}$-di-naphthalen-1-yl-biphenyl4,4'-diamine (TNB) as the HTL material and bis(10-hydroxyben-zo[h]quinolinato) beryllium (Bebq2) as the ETL material. The mobility of TNB and Bebq2 is at the same order of magnitude from our time of flight (TOF) measurement. Therefore, a device with more balanced carrier transport leads to better device performance. At 10 $\mathrm{mA} / \mathrm{cm}^{2}$, the drive voltage of the devices is as low as $3.16 \mathrm{~V}$ since the use of the high mobility ETL, Bebq2. The voltage variation when changing HTL thickness is nearly the same as that when changing ETL thickness. That shows the voltage drop is higher on HTL than ETL due to the use of high mobility ETL material. That also leads to the more balanced carrier transport than that in a conventional OLED. We also observed that a thinner device has longer operation lifetime that may be due to fewer traps in such a device.
\end{abstract}

Keywords: OLED, balanced charge transport

\section{INTRODUCTION}

Recently, the study of organic light-emitting device (OLED) has raised many attentions both in scientific society and commercial industry due to its advantages such as flexibility [1], light-weight, self-emitting, wide view-angle and the potential of low cost. Such a device is driven by electric current and light emission from amorphous organic films, which was first observed and extensively studied in 1960s [2],[3]. A two-layer device structure was introduced in 1987 combined with thermal evaporation technique in vacuum for thin film deposition to give lower bias voltage and better emission efficiency [4],[5]. Typically, OLED is constituted by a transparent conductive anode, a hole transport layer (HTL), an electron transport layer (ETL) and the metal cathode. A hole injection layer (HIL) and an electron injection layer (EIL) can be added to improve the injection of the electrical carrier. The carriers are injected from the anode and the cathode and then transport through the HTL and ETL with the hopping mechanism, respectively. Electrons and holes recombine when they reach the HTL/ETL interface, forming exciton and then emit photons. The light-emitting process of OLED is similar to the inorganic LED. However, there is a major difference between OLED and LED. Carrier mobility of an organic material is usually much lower than that of a semiconductor. In OLED, the HTL materials usually exhibit higher carrier mobility than ETL materials [6],[7]. Such an unbalance in carrier mobility means the amount of electrons and holes at the emission zone is different that leads to low current efficiency in device performance. Once the charge transport is unbalanced, the holes may penetrate into the emitting layer (EML) as cations that will form quenchers after long term operation [8],[9].

Here, we select $\mathrm{N}^{4}, \mathrm{~N}^{4}$-Di-naphthalen-2-yl- $\mathrm{N}^{4}, \mathrm{~N}^{4}$-di-naphthalen-1-yl-biphenyl-4,4'-diamine (TNB) as the HTL material and bis(10-hydroxyben-zo[h]quinolinato) beryllium (Bebq2) as the ETL material. Molecule structures of these two materials are shown in Fig. 1. Fig. 2 shows the Carrier mobility values of some organic materials with time of flight (TOF) measurement. Except TNB and Bebq2, N,N -bis(naphthalen-1-yl)-N,N -bis(phenyl) benzidine (NPB) and tris(8hydroxy-quinoline)aluminum (Alq3) those are commonly used HTL and ETL materials were also shown [10], [11]. We

*oeoled@yahoo.com.tw; phone 886223635251 ext. 125; fax 8862 2367-7467 
can see that NPB exhibits a hole mobility that is about six times higher than the electron mobility of Alq3. That causes the carrier unbalance in a conventional OLED device. However, mobility values of TNB and Bebq2 is at the same order of magnitude from our time of flight experiment. Therefore, a device with more balanced carrier transport leading to better performance and longer lifetime can be expected.

In this paper, we present the performances of five devices with different HTL and ETL thickness. Driving voltages of these devices are lower than conventional ones since we use the high mobility ETL material. Under the same current density, we found that the value of voltage increase with increasing HTL thickness is nearly the same as that in varying ETL case. That means the voltage drop on the HTL is higher than that on the ETL. Such a result is quite different from the conventional OLED. It also leads to more balanced transport characteristics in our device. We also found the thinner devices exhibit higher lifetime than thicker devices. The experimental details are represented in section 2 . The results and the related discussions are shown in section 3. Conclusions are drawn in section 4.
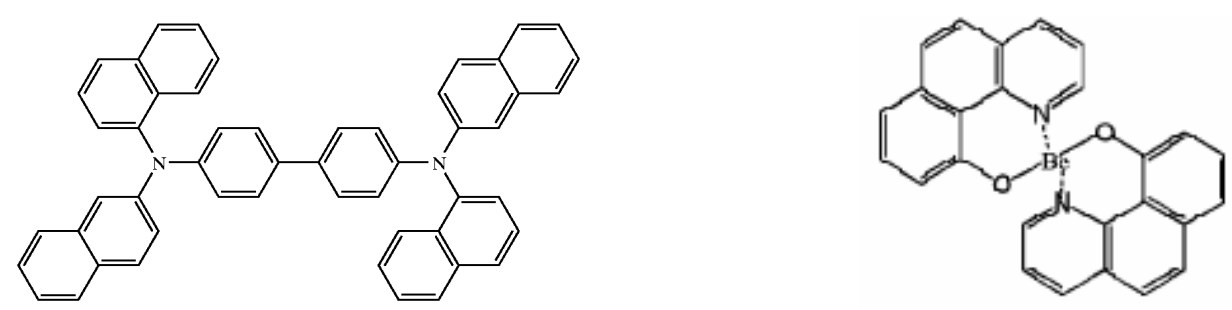

Fig. 1 The molecular structures of TNB (left) and Bebq2 (right).

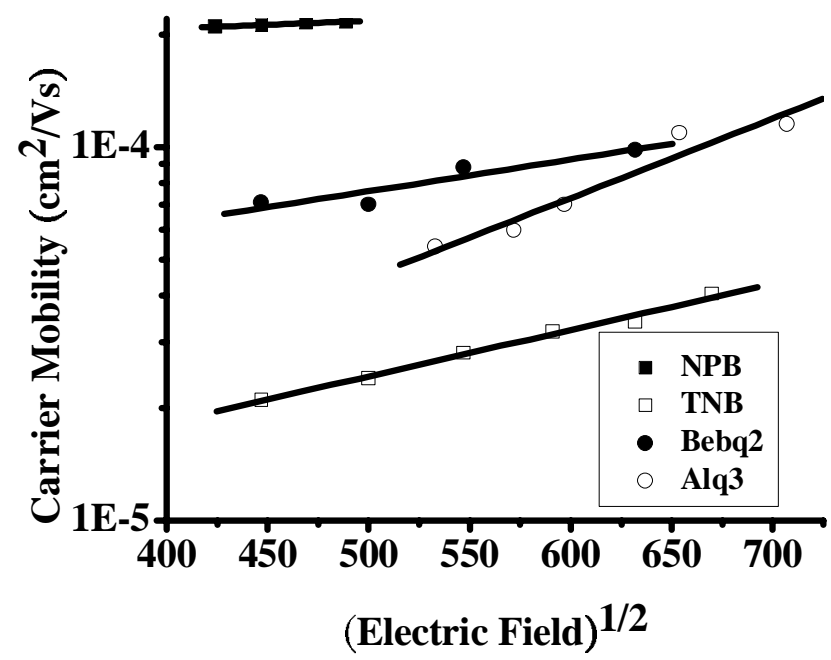

Fig. 2 Carrier mobility of NPB, TNB, Alq3 and Bebq2 measured by TOF method.

\section{EXPERIMENTS}

All our devices are prepared in multi-chamber cluster evaporator under the pressure about $1 \times 10^{-7}$ torr. The substrate is indium-tin-oxide (ITO) glass with sheet resistance $10 \mathrm{Ohm} / \mathrm{sqr} . \mathrm{O}_{2}$ plasma treatment was used to clean the ITO substrate surface and to improve the ITO work function before the deposition. The deposition rates of organic materials are typically $0.1 \mathrm{~nm} / \mathrm{sec}$. After evaporative deposition in the vacuum chamber, the devices were transported to the glove box with $\mathrm{O}_{2}$ and $\mathrm{H}_{2} \mathrm{O}$ concentration below $1 \mathrm{ppm}$. Encapsulation was completed in the glove box. Desiccant was used to absorb moisture that leak into the device. The active pixel size of a device is $0.5 \mathrm{~mm}$ x $0.5 \mathrm{~mm}$. We used Keithley 2400 source meter I-V characteristics. Minolta CS-1000 photometer was used to measure the luminance, the spectrum, and the CIE coordinate of the device. Lifetime was measured under the DC constant current of the initial brightness of 
$10000 \mathrm{~cd} / \mathrm{m}^{2}$ and recorded every 5 minutes. All these measuring instruments were connected to the personal computer and controlled by Labview programs.

In our devices, we use TNB as HTL and Bebq2 as ETL materials, respectively. ETL also serves as the emitting layer (EML). A thin layer of lithium fluoride ( $\mathrm{LiF}$ ) is inserted between the electron transport layer and the aluminum metal cathode to help improve the injection of the electron from cathode to ETL. The thickness of the aluminum cathode was $100 \mathrm{~nm}$. Device structures are shown in Table 1. By comparing the devices 1, 2, and 3, we can see the effects of changing the thickness of the hole transport layer. By comparing the devices 4, 2, and 5, we can see the effects of increasing the thickness of the electron transport layer. Device 1 (2) and 4 (5) exhibits the same thickness of total organic layers. It can be used to compare the effects between these two materials.

\begin{tabular}{|c|c|c||c|c|}
\hline sample & HTL (TNB) & ETL (Bebq2) & LiF & Al \\
\hline Device 1 & 600 & 600 & 7 & 1000 \\
\hline Device 2 & 800 & 600 & 7 & 1000 \\
\hline Device 3 & 1000 & 600 & 7 & 1000 \\
\hline Device 4 & 800 & 400 & 7 & 1000 \\
\hline Device 5 & 800 & 800 & 7 & 1000 \\
\hline
\end{tabular}

Table 1 Device structure (unit: ̊)

\section{RESULTS AND DISCUSSIONS}

The resulting J-V curve is shown in Fig. 3. It indicates that at constant current density, the drive voltage is higher when increasing device thickness. When increasing HTL thickness, the drive voltage increases more severely than that when increasing ETL thickness. At $10 \mathrm{~mA} / \mathrm{cm}^{2}$, the drive voltage of the devices is $3.11 \mathrm{~V}$ (device 1 ), $3.63 \mathrm{~V}$ (device 2 ), and $4.44 \mathrm{~V}$ (device 3 ) when varying HTL thickness from $600 \AA$ to $1000 \AA$ with fixed ETL thickness at $600 \AA$. At the same current density, the drive voltage is $3.12 \mathrm{~V}$ (device 4), 3.63V (device 2), and 4.08V (device 5) when varying ETL thickness from $400 \AA$ to $800 \AA$ with fixed HTL thickness $800 \AA$. The voltage variation when changing HTL thickness is nearly the same as that when changing ETL thickness. Compared with previous results [12], we can see the driving voltage in our device is smaller than that in a conventional device. That is due to the use of high mobility ETL, Bebq2. Since the driving voltage variation when varying HTL and ETL thickness is comparable, it is the evidence that the charge is balanced in our device. Although the total thickness of organic layer in device 1 and device 4 are the same, the drive voltage of device 1 at $10 \mathrm{~mA} / \mathrm{cm}^{2}$ is lower than that of device 4 . The difference between these two devices is that the HTL (ETL) thickness of device 4 is $200 \AA$ larger (smaller) than device 1 . That results from the low hole mobility of TNB and high electron mobility of Bebq2. The same phenomenon exists when comparing device 3 and device 5 . That means, the voltage drops on the HTL is higher than that on ETL in our devices. 


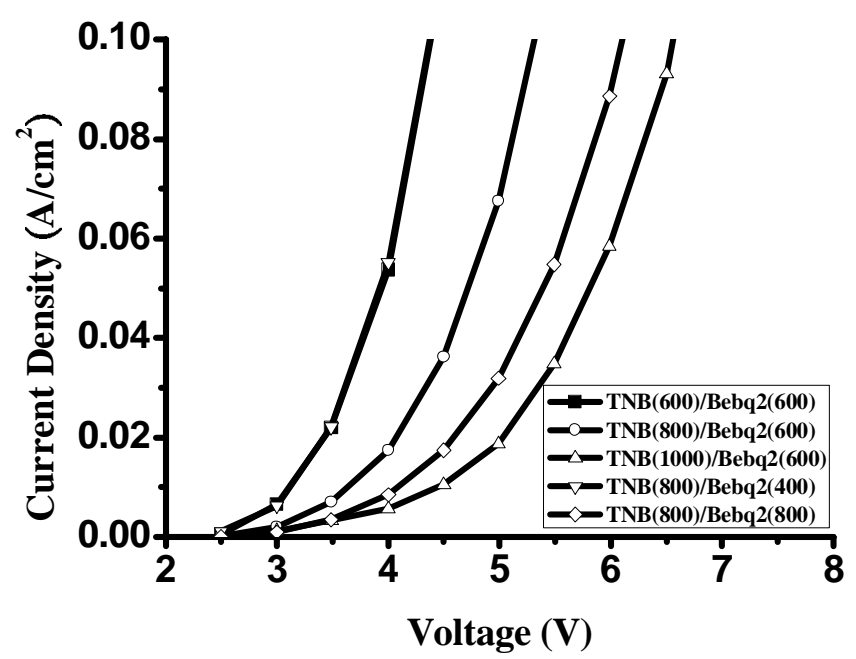

Fig. $3 \mathrm{~J}-\mathrm{V}$ curves of the different devices.

Fig. 4 shows the B-J curves of the five experimental devices. Fig. 4(a) is the group with different HTL thickness and the fixed ETL thickness. The current efficiencies at $100 \mathrm{~cd} / \mathrm{m}^{2}$ of the devices are $5.64 \mathrm{~cd} / \mathrm{A}$ (device 1), 5.21 cd/A (device 2), and $5.16 \mathrm{~cd} / \mathrm{A}$ (device 3 ). We can see that thicker HTL has lower brightness at constant current density, i.e. lower current efficiency. Since the hole mobility of TNB is small than the electron mobility of Bebq2, thicker TNB means more unbalanced in our experiments that results in the low current efficiency. Fig. 4(b) is the group with different ETL thickness and fixed HTL thickness. The current efficiencies at $100 \mathrm{~cd} / \mathrm{m}^{2}$ of the devices are $3.27 \mathrm{~cd} / \mathrm{A}(\mathrm{device} 4)$, $5.21 \mathrm{~cd} / \mathrm{A}$ (device 2), and $5.55 \mathrm{~cd} / \mathrm{A}$ (device 5). When ETL thickness is varied from $600 \AA$ to $800 \AA$, the current efficiency of these two devices is almost the same. That means the amount of electrons is larger than that of holes at the HTL/ETL interface hence the current efficiency is dominated by the HTL thickness. However, when ETL thickness is decreased to $400 \AA$, the current efficiency has a rapid decrease that is due to the optical destructive interference from the cathode.

(a)

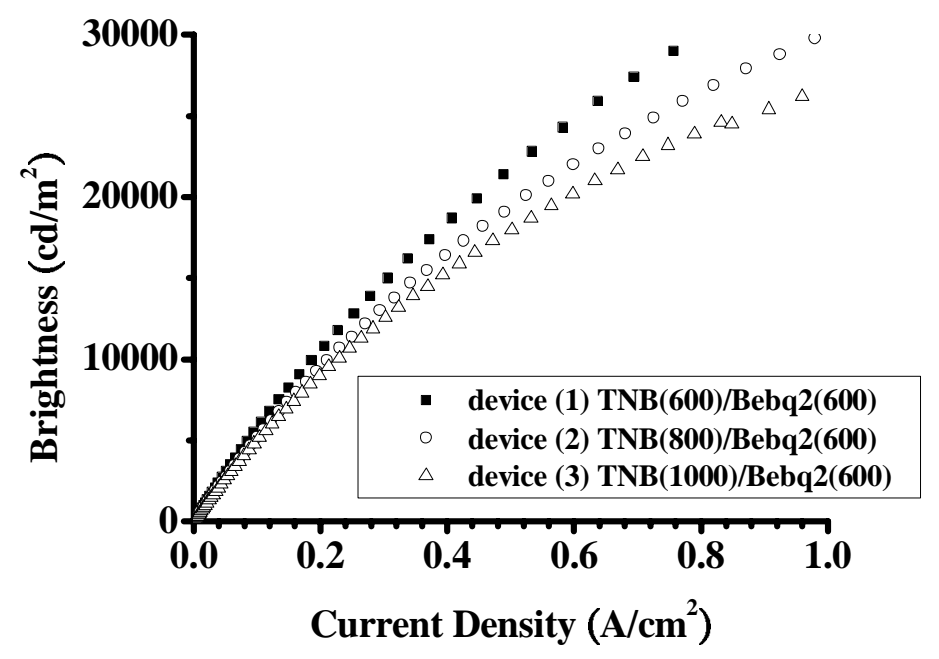


(b)

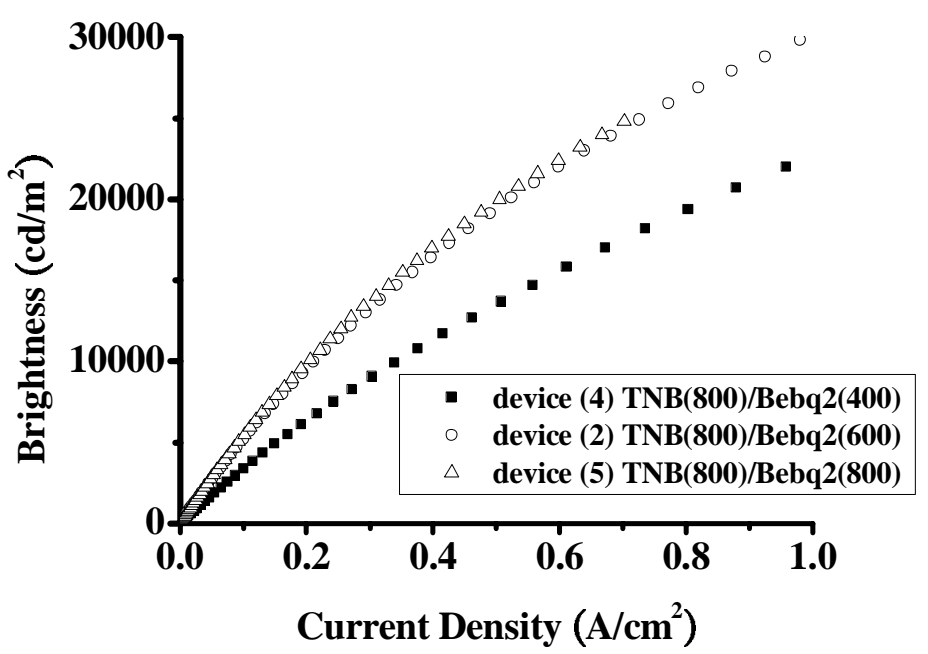

Fig. 4 (a) Brightness-current density curve of the devices 1, 2, and 3. (b) Brightness-current density curve of the devices 4, 2, and 5.

Accelerated lifetimes of devices are shown in Fig. 5. When varying HTL thickness, the accelerated half-lifetimes are $6.08 \mathrm{hr}$ (device 1), $2.33 \mathrm{hr}$ (device 2), and $1.83 \mathrm{hr}$ (device 3 ) with initial brightness at $10000 \mathrm{~cd} / \mathrm{m}^{2}$. And when varying ETL thickness, the lifetimes are $3.61 \mathrm{hr}$ (device 4), 2.33hrs (device 2), and $2.33 \mathrm{hr}$ (device 5) with initial brightness at $10000 \mathrm{~cd} / \mathrm{m}^{2}$. When varying HTL and ETL thickness, we can see that thinner devise exhibits longer lifetime. It has been shown that the degradation of OLED comes from the quencher formation in emitting layer (EML) [13]. There are fewer traps in a thinner device and hence it exhibits a longer lifetime. In our device, Bebq2 is used as ETL and EML that exhibits low current efficiency as compared with Alq3. An additional EML should be inserted between HTL and ETL for longer operation lifetime. For the devices with the same organic layer thickness, we can see that device with more balanced carrier transport characteristic has a longer lifetime, i.e. device 1 (5) has longer lifetime than device 4 (3).

(a)

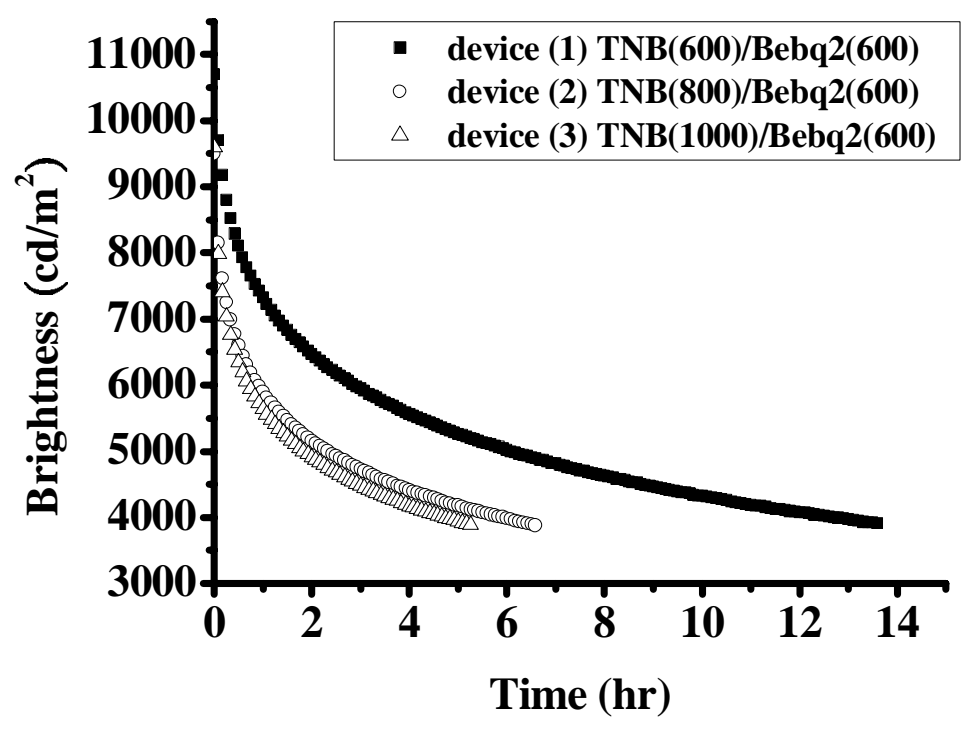


(b)

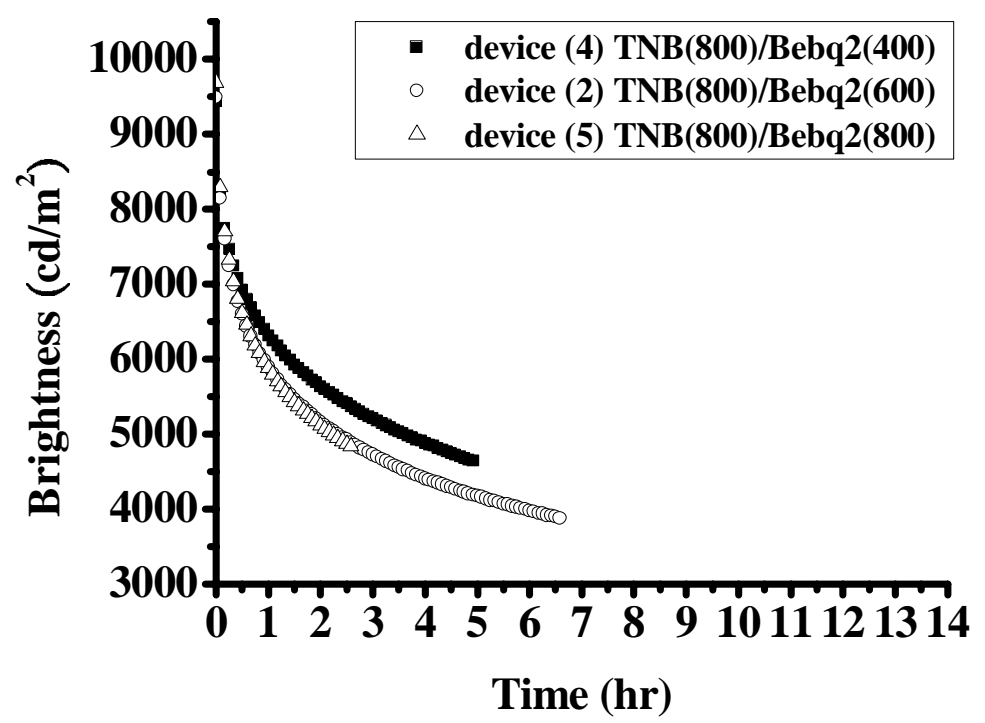

Fig. 5 (a) The lifetime of the devices 1,2, and 3. (b) The lifetime of the devices 4, 2, and 5.

\section{CONCLUSIONS}

From the experimental results shown above, we can see that the charge transport is more balanced in our device. Unlike the conventional HTL and ETL materials, we select TNB and Bebq2 as the HTL and ETL material whose hole and electron mobility are at the same order of magnitude. Bebq2 exhibits a high mobility and hence decrease the driving voltage. Devices with balanced carrier transport exhibits higher current efficiency. Although the balanced charge transport is important for operating lifetime of OLED, another crucial element is the thickness of the organic layer. Thicker organic layer leads to shorter operational lifetime due to more traps in such a device.

\section{ACKNOWLEDGEMENT}

This work was supported by the National Science Council, the Republic of China, under Grant No. NSC 92-2218-E002-050.

\section{REFERENCES}

1. M. S. Weaver, L. A. Michalski, K. Rajan, M. A. Rothman, J. A. Silvernail, and J. J. Brown, Appl. Phys. Lett. 81, 2929 (2002).

2. E. Gurnee, R. Fernandez, US Patent 3172862 (1965).

3. M. Pope, H. Kallman, P. Magnante, J. Chem. Phys. 38, 2042 (1963).

4. C.W. Tang, US Patent 4356429 (1982).

5. C.W. Tang, S.A. VanSlyke, Appl. Phys. Lett. 51, 913 (1987).

6. Baijun CHEN, Chun-sing LEE, Shuit-tong LEE, Jap. J. Appl. Phys. 39, 1190 (2000).

7. Shigeki Naka, Hiroyuki Okada, Hiroyoshi Onnagawa, Synth. Metals 111-112, 331 (2000).

8. D. Y. Kondakov, J. R. Sandifer, and C. W. Tang, J. Appl. Phys. 93, 1108 (2003).

9. Z. D. Popovic, H. Aziz, N.-X. Hu, A. Ioannidis, and P. N. M. dos Anjos, J. Appl. Phys. 89, 4673 (2001).

10. Zhenbo Deng a,), S.T. Lee a, D.P. Webb, Synth. Metals 107, 107 (1999).

11. R. G. Kepler,a) P. M. Beeson, S. J. Jacobs, Appl. Phys. Lett. 66, 3618 (1995).

12. Jingsong Huang, Jan Blochwitz-Nimoth, Martin Pfeiffer, J. Appl. Phys. 93, 838 (2003).

13. Wolfgang Brütting, et. al., Device physics of organic light-emitting diodes based on molecular materials, Organic Electronics 2, 1 (2001). 